\title{
Hein Retter (Deutschland) \\ Zur Frage der Toleranz in der Epoche zwischen Reformation und Aufklärung
}

\begin{abstract}
Summary (Hein Retter: On the Question of Tolerance in the Epoch Between the Reformation and Enlightenment): This article deals with the origins of religious tolerance in the modern era. It goes back to the early modern era, when intolerance by the Roman-Catholic church towards new reformative movements showed itself to be particularly pervasive. At the same time, the Roman-Catholic church faced opposition from regional princes and free imperial cities who had become powerful and frequently tended to lean towards the new faith. They demanded the acknowledgment of the reformative faith by the pope and the emperor. However, they could hardly be called tolerant towards other faiths in their own territories, especially in the case of minorities seeking public recognition of their alternative beliefs and religious practices. Stark intolerance eased off only when tolerance functioned as an inherent political necessity, in hopes of gaining large economic benefits, especially under secular rule yet hardly ever under that of the church. The results from an international conference presented here show that tolerance in the age of the Reformation cannot be confused with the mutual recognition of religious and cultural idiosyncracies, in the way these are often claimed nowadays when advocating for a peaceful coexistence of different groups in a pluralistic society. In the historical context of the early modern era, tolerance was a one-sided act-in hopes of political and economic advantages - towards gaining a kind of freedom which, in its overall effect, definitely involved risks of conflict. In this context, differing political structures such as the personal beliefs of the ruling prince influenced the different climates regarding tolerance in 16th-to 19th-century Europe.
\end{abstract}

Keywords: Tolerance, intolerance, church, Reformation, early modern era, confessional era

Резюме (Хейн Реттер: К вопросу толерантности в эпоху между Реформацией и Просвещением): Статья рассматривает происхождение толерантности в Новое время. При этом он обращается $к$ раннему периоду Нового времени, когда особенно глубоко проявилась нетерпимость римско-католической церкви по отношению к новым реформаторским течениям. Одновременно римско-католической церкви противостояли ставшие сильными, в большинстве своем склоняющиеся к новой вере, удельные князья и свободные имперские города. Они требовали от Папы и императора признания реформаторской веры. Но на их собственной территории, исходя из их отрицательного отношения к инаковерующим, их, вряд ли, можно было назвать толерантными, в частности, если меньшинство искало общественного признания их отличающегося вероисповедания и их религиозной практики. Лишь если благодаря получению больших экономических выгод толерантность действовала в политике, так сказать, в качестве делового нажима, то жесткая непримиримость ослаблялась - но это, прежде всего, под воздействием светской, но не церковной власти. Проведенный для этого анализ результатов международной конференции показывает, что толерантность в эпоху Реформации нельзя смешивать со взаимным признанием религиозного и культурного своеобразия, как это сегодня часто требуют, выступая за мирное сосуществование различных групп в плюральном обществе. В историческом контексте раннего периода Нового времени толерантность была односторонним - ожидающим политических преимуществ - актом предоставления свободы, которая в своем совокупном воздействии непременно предполагала риски конфликтов. При этом различные политические структуры, как и личные убеждения правящих князей непременно оказывали влияние на различия климата толерантности, встречающихся в Европе в 16-м - 18-м веках.

Ключевые слова: толерантность, нетерпимость, церковь, Реформация, ранний период Нового времени, конфессиональная эпоха 
Zusammenfassung: Der Beitrag behandelt die Ursprünge religiöser Toleranz in der Moderne. Er geht dabei auf die frühe Neuzeit zurück, als sich die Intoleranz der römisch-katholischen Kirche gegenüber neuen reformatorischen Strömungen besonders nachhaltig zeigte. Gleichzeitig standen der römischkatholischen Kirche mächtig gewordene, vielfach zum neuen Glauben tendierende Territorialfürsten und freie Reichsstädte gegenüber. Sie forderten die Anerkennung des reformatorischen Glaubens von Papst und Kaiser. Auf eigenem Gebiet konnte man sie in ihrer negativen Haltung gegenüber Andersgläubigen allerdings kaum tolerant nennen, insbesondere wenn Minderheiten um öffentliche Anerkennung ihres abweichenden Bekenntnisses und ihrer religiösen Praxis nachsuchten. Nur wenn durch Gewinnung großer ökonomischer Vorteile Toleranz gleichsam als Sachzwang in der Politik fungierte, lockerte sich starre Intoleranz auf - dies vor allem unter weltlicher, kaum aber unter kirchlicher Herrschaft. Die hierzu vorgenommene Auswertung der Befunde einer internationalen Tagung ergibt, dass Toleranz im Zeitalter der Reformation nicht verwechselt werden darf mit wechselseitiger Anerkennung religiöser und kultureller Eigenheiten, wie sie heute im Eintreten für das friedliche Zusammenleben verschiedener Gruppen in einer pluralen Gesellschaft oft gefordert wird. Im historischen Kontext der frühen Neuzeit war Toleranz ein einseitiger - politische oder ökonomische Vorteile erhoffender - Akt der Gewährung einer Freiheit, die in ihrer Gesamtwirkung durchaus Konfliktrisiken beinhaltete. Dabei besaßen differente politische Strukturen wie persönliche Überzeugungen des regierenden Fürsten durchaus Einfluss auf die in Europa im 16 bis 18. Jahrhundert anzutreffenden Unterschiede des Toleranzklimas.

Schlüsselwörter: Toleranz, Intoleranz, Kirche, Reformation, Frühe Neuzeit, konfessionelles Zeitalter

\section{Einführung}

Der moderne Begriff der Intoleranz steht wie sein Gegenstück „Toleranz“ in engem Bezug zur christlichen Religion und ihrer Geschichte. Die drei großen, im Nahen Osten entstandenen großen monotheistischen Religionen - Judentum, Christentum und Islam - waren in vormodernen Epochen intolerant. Alle drei Religionen gehen davon aus, dass nur ein einziger Gott, nämlich der ihre, die Wahrheit und das Heil verkörpert. Die Bibel zeigt dies in vielen ihrer Erzählungen für das antike Judentum, selbst wenn es Ausnahmen gibt (wie das Buch Rut); das rabbinische Judentum gewann in seiner geographischen Zerstreutheit Universalität, öffnete sich aber auch gegenüber Kulturen Einflüssen. Das Merkmal religiöser Intoleranz, verstanden als heiliger Krieg gegen Ungläubige, ist im Koran zu finden. Die Geschichte des Christentums ist voll von Auseinandersetzungen, in denen es um die Durchsetzung der in Dogmen der Kirche verankerten Glaubenswahrheit geht, dass allein Jesus Christus der Erlöser sei, der Anspruch auf Gefolgschaft habe.

Man könnte meinen, Luther und die Reformation habe der bestehenden Intoleranz der Kirche als europäischer Großmacht ein Ende gesetzt. Weit gefehlt! Luther zeigte sich hinsichtlich religiöser Anschauungen, soweit sie abwichen von der eigenen Auffassung, besonders intolerant. Aber seine Anhänger waren, wie er selbst unter Friedrich dem Weisen, auf die Toleranz ihres Landesherrn angewiesen. Falls der Fürst katholisch blieb, hatten sie Vertreibung oder Schlimmeres zu befürchten. Auf Gebieten des lutherischen und des calvinistischen Glaubens ging es, grob gesprochen, kaum weniger intolerant zu als in katholischen. Es wird zu klären sein, wie in der frühen Neuzeit im Zeitalter von Renaissance, Humanismus und Reformation das schwache Pflänzchen „Toleranz“ Wurzeln fasste und was wir heute daraus lernen können.

Vom 22. bis 24. Juli 2013 fand am Gothaer Forschungszentrum für kultur- und sozialwissenschaftliche Studien eine internationale Tagung statt zur Entstehung des modernen Toleranzbegriffs in der frühen Neuzeit. Der nun vorliegende Tagungsband beinhaltet - neben einer Einführung - die fünfzehn in deutscher und englischer Sprache wiedergegebenen Tagungsreferate. Sie sind folgenden Themengruppen zugeordnet (in Klammern die Namen der Referenten):

- Grenzen der Toleranz (Jan Assmann, Walter Sparn, Lukasz Bienasz); 
- Dissidenten und Randgruppen (Kestutis Daugirdas, Sascha Salatowsky, Justin Champion; Friedrich Vollhardt);

- Nationale Varianten des Toleranzdiskurses (Mona Garloff, Jens Glebe-Møller, John-Christian Laursen, Wiep van Bunge, Ulrich Niggemann);

- Juristische und sozialgeschichtliche Aspekte (Wolfgang Forster, Harald Stockert, Alexander Schunka).

Der Band gibt zu Überlegungen Anlass, die über eine Buchbesprechung weit hinausgehen. Gleichwohl verdient er eine Würdigung der Einzelbeiträge. Einen Autorenspiegel, der über Vita und Forschungsschwerpunkte der Referenten Auskunft gibt, enthält der Band leider nicht. Zehn Jahre nach Erscheinen des historisch-systematischen Standardwerkes von Rainer Forst „Toleranz im Konflikt" (2003) sah sich die Gothaer Tagung vor die Aufgabe gestellt, nicht nur den Erkenntnisstand zur „Tolerantia“ im 16. bis 18. Jahrhundert zu markieren, sondern auch die Frage zu stellen, ob die Hochschätzung von Toleranz in den liberalen Demokratien westlicher Prägung, ebenso in liberalen Religionsgemeinschaften, heute für die Erforschung ihrer Geschichte nicht eher ein Hindernis bildet, wenn vorurteilsfreies historisches Urteilen erwünscht ist. In der Regel steht das moralische Urteil mit seiner dichotomen Struktur von Gut und Böse einem um Differenzierung bemühten historischen Urteil im Weg. Ob die Moral über die Geschichte den Sieg davon getragen hat, zeigt sich am Grad historischer Mythenpflege, mit der gute Toleranz und böse Intoleranz zu Werkzeugen werden, die Geschichte der Toleranz zu schreiben, die dann sehr viel mehr idealisierte (oder janusköpfige) Moralgeschichte als komplexe Realgeschichte darstellt. Dieser Beziehungsfalle auszuweichen war Anliegen der Tagung.

Im Vorwort der Herausgeber - Sascha Salatowsky leitet in Gotha das Forschungszentrum für kultur- und sozialwissenschaftliche Studien der Universität Erfurt, Winfried Schröder lehrt Geschichte der Philosophie an der Universität Marburg - wird dies deutlich. Die Forschung habe zwar einen erfreulichen Aufschwung genommen, doch gleichzeitig zu einem „Klärungs- und Vertiefungsbedarf“ geführt. Ein solcher Bedarf ergibt sich aus mehreren Gründen, sei es, dass der Blick der Historiker zu sehr vom Kanon „der als Meilensteine der einschlägigen Debatten eingestuften Texte“ etwa Spinozas, Lockes, oder Bayles gefangen genommen wurde, sei es, dass Humanismus, Reformation und Aufklärung im Zentrum des vorherrschenden Forschungsinteresses standen, ohne dass dabei den epochalen Zwischenphasen, einzelnen Territorien mit besonderem Konfliktpotential oder manchen Gruppen religiös Verfolgter hinreichende Aufmerksamkeit geschenkt worden sei. Drittens aber, und das macht den Band besonders interessant, stellten die Veranstalter die Frage: Gab es in der frühen Neuzeit in Europa nicht auch ein legitimes Motiv, religiöse Toleranz als Übel und somit Intoleranz als einzig mögliche Konsequenz zum Prinzip von Herrschaft und sozialer Ordnung zu erheben?

\section{Cura religionis - die Sorge um die Religion als Glaubenszwang}

Dieses Motiv existierte in der Tat. Es war die cura religionis, die „Sorge um die Religion“, wie Winfried Schröder in seinem Einführungsbeitrag klar macht. Allerdings trat diese Sorge immer auch vermischt mit anderen Motiven auf. Gewann der Begriff vor allem in den protestantischen Territorialstaaten kirchenrechtliche Bedeutung durch die Zuständigkeit des Landesherren für alle kirchlichen Angelegenheiten, so wird cura religionis von den Herausgebern des Bandes in einem erweiterten, zeitübergreifenden Sinn verstanden. Gemeint ist ganz allgemein die Sorge um Sicherheit und Frieden für die Menschen. 
Im christlichen Glaubensleben hatte sich die Sorge um die Religion als Glaubenszwang etabliert, seit das Christentum unter Kaiser Theodosius I. ab 380 n. Chr. Staatsreligion des Römischen Reiches geworden war. Die Religionsfreiheit, die das Mailänder Edikt 313 n.Chr. gewährte, wurde damit beseitigt. Kaiser Konstantin I., ab 324 Alleinherrscher im Römischen Reich, besaß pragmatische Toleranz. Er privilegierte die Christen als kleine religiöse Elite, ohne zugleich die Masse des Heidentums zu bekämpfen. Verfügten doch die Bischöfe im Reich über eine hervorragende Infrastruktur, die angesichts der nur schwach ausgeprägten römischen Verwaltung politisch zu nutzen war. Paul Veyne (Als unsere Welt christlich wurde. Aufstieg einer Sekte zur Weltmacht, 2008, S. 19f.) erinnert daran, dass das Schicksal des Christentums in den Jahrzehnten nach Konstantins Tod (†337) im Kreis der Religionen des mediterranen Raumes, politisch gesehen, am seidenen Faden hing, und seine spätere Weltmachtstellung keineswegs voraussehbar war. Der Kampf der Kirche gegen Häresie im Inneren war so kompromisslos wie der Kampf gegen alle religiösen Überzeugungen, die ihre Macht von außen bedrohten. Tertullians Wort, dass außerhalb der Kirche kein Heil zu finden sei, und die Überzeugung Augustins, es geschehe um des Heils der Menschen willen, wenn sie gegebenenfalls zwangsweise christianisiert werden, beherrschten die großen christlichen Konfessionen Europas noch in der Missionierung nichteuropäischer Völker mit ihrem Höhepunkt im Kolonialzeitalter bis zum Ersten Weltkrieg.

Schröder erwähnt Augustins Rechtfertigung, Bekehrungsunwillige notfalls mit Zwang zum christlichen Glauben zu verhelfen. Der Kirchenvater verwies auf das Wort Jesu, „Compelle intrare“ (Nötige sie einzutreten!) aus dem Gleichnis vom großen Gastmahl (Lk 14,23): Die Kirche habe dafür Sorge zu tragen, dass möglichst viele bewahrt werden vom Schicksal ewiger Verdammnis.

Winfried Schröder betont, dass die Argumentation Augustins nicht Ausdruck von Zynismus war. Der Kirchenvater habe sich nicht der Illusion hingegeben, allein durch Zwang Glauben aus freien Stücken zu erreichen, Belehrung sei ebenso nötig. Augustinus sah im Zwang, so Schröder, ein Mittel, Nichtgläubige zu nötigen, ihren Standpunkt zu überdenken, damit sie sich dem Christentum als der allein selig machenden Religion zuwenden. Wer nicht der "wahren Lehre" folgte, musste mit ewiger Verdammnis rechnen, so bezeugt es das Neue Testament.

\section{Die christliche Delegitimierung jüdischer Heilserwartung}

Christen übernahmen den Exklusivitätsanspruch, von Gott auserwählt zu sein, vom antiken Judentum. Unter römischer Besatzung leidend, war das Volk Israel von der apokalyptischen Hoffnung erfüllt, mit dem Erscheinen des erwarteten Messias (des „Gesalbten“, griech. Christos) werde ein neues Zeitalter in Frieden und Freiheit durch das irdisch-himmlische Königreich mit seinem Zentrum Jerusalem anheben, wie es die Bibel beschreibt (Offb 21,1-6). Dass der Messias in Gestalt des Juden Jesus von Nazaret bereits aufgetreten und nach seiner Kreuzigung zum Leben wiederauferweckt worden sei, lehrte der aus der Diaspora stammende Jude Paulus; er war durch eine Vision zu seiner Missionstätigkeit gekommen. Das fromme Judentum widersetzte sich seiner Lehre.

Paulus' Theologie formte den christlichen Glauben, indem er das Judentum theologisch als Bündnispartner Gottes delegitimierte: Der Tempel, Jerusalem als Ort der Erfüllung messianischer Hoffnung und die Erfüllung der Gesetze gehörten zum Kern jüdischer Frömmigkeit. Davon hatte sich die Mission des Paulus deutlich distanziert. Das Christentum entsprang dem antiken Judentum, doch es wurde von Paulus mit deutlicher Distanz zum Judentum gelehrt (einschließlich seiner Distanz zu den Jüngern Jesu als den Leitern der Jerusalemer Urgemeinde). Zum einen tat dies Paulus pragmatisch, indem der neue Messiasglaube nicht unnötig Anstoß erregen sollte, zum andern theologisch durch die Einführung der Differenz von der Bindung an das Gesetz (Judentum) und der Freiheit des 
Evangeliums (Christentums), drittens emotional durch die Zuordnung des überwiegend unbelehrbaren Judentums zu Gegnern, die Paulus dem Zorn Gottes ausgeliefert sah (1Thess 2,14-16). Die Juden in Korinth, die ihm verständlicherweise verwehrten, in der Synagoge vom Messias zu predigen, verfluchte Paulus: „Euer Blut komme über euer Haupt“ (Apg 18,6). Das Gebot Jesu „Liebet eure Feinde!" (Mt 5,44) bezog Paulus, dem Jesus als Prediger nie begegnet war, offenbar nicht auf Glaubensfeinde. Paulus' Lehre bildete den Anfang des theologischen wie emotionalen Antijudaismus im Christentum. Soweit meine Vorüberlegungen.

Jan Assmann verweist in seinem Referat über „Monotheismus und Intoleranz" darauf, dass die jüdische Religion aus zwei differenten Ursprungsmythen hervorging: dem friedlichen Erzväter-Mythos, der Israel zum Bundespartner Gottes machte, und dem von Mose geführten Auszug aus Ägypten, der mit der Landnahme Kanaans ein ungeheures Maß an Gewalttaten einschloss. Die Geschichte Israels, die Kernbestand seiner Religion ist, ist - auch - eine Geschichte der Gewalt. Sei es, dass Israel als das erwählte Volk den „Feinden Gottes“ mit Gewalt begegnete, sei es, dass das politisch schwache jüdische Volk durch starke Feinde Besetzung, Asylierung und schweres Leid zu erdulden hatte. Mit dem Exodus-Mythos war eine identitätsbildende, das Bewusstsein Israels prägende Sonderstellung gegenüber den anderen Völkern gegeben. Nicht die Gewaltbereitschaft, sondern der religiöse Exklusivismus, in dessen Dienst die Gewalt ausgeübt wurde, ist das Besondere.

Der Monotheismus des antiken Judentums beinhaltet nach Assmann zwei Aspekte: Die strenge Unterscheidung zwischen Gottesfürchtigen und Gottesleugnern geht zurück auf die Dichotomie der „Wahrheit" des einzigen Gottes und der „Falschheit“ der vielen Götzen, die die Feinde Israels anbeteten. Freund und Feind, Wahrheit und Falschheit sind in den heiligen Schriften genau bezeichnet. Der Tanach, die hebräische Bibel, ist die kodifizierte Offenbarung des göttlichen Willens, den das Volk durch Befolgung der Gesetze zu erfüllen suchte. Im Bewusstsein der IHM geschuldeten Bundestreue hatte Israel dem Gesetz unbedingten Gehorsam zu leisten. Zugleich blieb bei jahrhundertelang andauernder politischer Abhängigkeit die Erwartung künftigen Heils lebendig. Diese Konstellation stellte, so Assmann, etwas durchaus Einzigartiges in der Antike dar. Sie legitimierte den heiligen Krieg unter Berufung auf das Gesetz, das für Israel im Verweis auf Gottes Wort in der Schrift absolute Geltung besaß. Die exklusive Idee des Heils, dass Jesus von Nazaret der Christus sei, nahm das Christentum für sich in Anspruch. Andere Völker durften an diesem Heil teilhaben. Dass dabei nicht die Reinheit der christlichen Religion beschädigt wurde, darüber wachte die Kirche mit strenger Intoleranz.

\section{Religiös motivierte Gewalt in den Glaubenskriegen der frühen Neuzeit}

In den religiösen Auseinandersetzungen ab dem 16. Jahrhundert waren Hass und Gewalt besonders ausgeprägt. Bis dahin hatte die Einheit der römisch-katholischen Kirche im System monarchischer Territorialstaaten und dem Sacrum Romanum Imperium wesentlich beigetragen zur politischen Stabilität Europas, trotz ständiger Auseinandersetzungen weltlicher wie kirchlicher Herrscher um Einfluss und Macht. Diese Einheit war mit den religiösen Aufbrüchen im dritten Jahrzehnt des 16. Jahrhundert überraschend schnell geschwunden. Der Abfall lutherischer und calvinischreformierter Glaubensrichtungen von der Kirche erzeugte eine religiöse Eigendynamik, die in ganz Europa um sich griff. Die Kämpfe um die „wahre“ Konfession waren von Verfolgung und Krieg bestimmt. Alle Versuche, die religiöse Einheit wiederherzustellen, zerschellten an der Etablierung des Konfessionalismus, der für die politisch erstarkten Territorialfürsten ein Medium erweiterter, in eigener Entscheidung getroffener religiöser Selbstbestimmung wurde und gegenüber Kaiser und Reich als Instrument der Politik zum Einsatz kam. Die römisch-katholische Kirche blieb dennoch, wie der Gang der Geschichte gezeigt hat, die stärkste religiöse Kraft in Europa. Die Dynamik des Neuen ging allerdings von den virulent auftretenden neuen religiösen Minderheiten aus. 
Von Toleranz gegenüber andersgläubigen Christen, Häretikern, konnte aus katholischer Sicht überhaupt nicht die Rede sein. Außerhalb des allgemeinen (katholischen) Glaubens durfte sich niemand Christ nennen. Wer dies tat, war ein Glaubensfeind, der zum „wahren" (katholischen) Glauben zu nötigen war um seines Seelenheils willen, andernfalls erwarteten den Unbelehrbaren durch die Inquisition Folter und Tod. Die katholische Kirche sah sich erst nach dem Zweiten Weltkrieg genötigt, die Auffassung zu korrigieren, religiöse Toleranz als Übel zu betrachten, dessen Duldung im Zeitalter verfasster Bürgerrechte sie notgedrungen hinnehmen musste. Erst mit dem II. Vatikanum 1965 wurde von ihr das Recht auf Religionsfreiheit voll anerkannt.

\section{Humanismus und Toleranz}

Überlegungen von Gelehrten der frühen Neuzeit, aus Gründen politischer Vernunft im Bedarfsfall eine begrenzte Duldung religiös Andersdenkender zuzulassen, gewinnen im Aufbruch von Renaissance und des Humanismus an Bedeutung. Die von Italien ausgehenden, ganz Europa erfassenden geistigen Impulse vermittelten in einer Epoche wirtschaftlicher Prosperität ein diesseitsgerichtetes Lebensgefühl, mit dem ein neues, auf die nicht-christliche Antike gerichtetes Bildungsinteresse einher ging. Bildung ermöglicht Aufklärung wie geistige Herrschaft. Die Bedeutung letzterer erkannte die Societas Jesu, der Jesuitenorden. Die Jesuiten machten die Erneuerung katholischer Bildung zur Speerspitze ihres Rekatholisierungsprogramms. Doch die Funktion der Selbstaufklärung der Menschen durch Bildung zeigte ihre Wirkungen in den reformierten Gebieten sehr viel stärker. Jeder sollte in der Lage sein, die Bibel in deutscher Sprache zu lesen. Grundbildung wurde zum Element des evangelischen Glaubens. Auf der Ebene der höheren Bildung schuf der Humanismus etwas Neues, das der christliche Glaube, dessen Urtugenden im Gehorsam gegenüber Gott, der Nächstenliebe und dem Halten der Gebote bestanden, so nicht kannte: Es wuchs ein neues Interesse für Dichtung, Philosophie, Literatur und Kunst der Antike, das über das universitäre Studium der tradierten septem artes liberales, der sieben freien Künste, weit hinausging. Es wuchs das Interesse für das Humanum, so wie es in der antiken Originalliteratur überliefert wurde. Das erforderte das Erlernen der alten Sprachen (Griechisch, Hebräisch) und die Kenntnis der alten literarischen Quellen sowie deren Übersetzung. Universale Sprachkenntnis, die über den zeitüblichen Gebrauch des Lateinischen als Gelehrtenssprache hinausging, erweiterte das Verstehen, stärkte die Achtung vor antiken - nichtchristlichen - Quellen und beflügelte neue Denkanstrenugnen zur Trennung faktischer Wahrheit von unwahren Behauptungen. Es mache erstmals die Einflüsse der griechischen Philosophie auf frühchristliches Denken sichtbar. Literarische Bildung ermöglichte eine gehaltvollere Kritik gegenüber sachlich unbegründeten Deutungsansprüchen, als sie die formalscholastische Kritik des Opponenten in der akademischen Disputationstradition bot. Das Interesse an den geschichtlichen Wurzeln der eigenen Vergangenheit erwachte; im deutschsprachigen Raum verband sich dieses Interesse auch mit gewissen, gegen Rom gerichteten nationalen Anklängen. Die Entdeckung neuer Länder, Völker und Erdteile, aber auch neue Theorien über den Kosmos und die Naturkräfte veränderten das Weltwissen, das dank des Buchdruckes weitergegeben werden konnte.

Die christliche Religion bildete dort, wo sie sich mit dem Humanismus von einzelnen, frei denkenden Gelehrten der Zeit verband, den Ausgangspunkt für Inseln toleranten Bewusstseins. Davon waren katholische Gelehrte, selbst Kirchenfürsten, nicht ausgenommen, wie die Friedensschrift von Kardinal Nikolaus von Kues von 1453 belegt. Toleranz zeigte der Humanist Johannes Reuchlin: Er hatte als Antwort auf die ihm von Kölner Dominikanern aufgezwungene Polemik 1511 empfohlen, Juden mit Toleranz zu begegnen. Die weithin bestehende Intoleranz der Zeit gegenüber dem Judentum bildete kein Thema der Gothaer Konferenz. Dass Reuchlin zu Luthers Aufbegehren gegen Papst und Kirche in Distanz stand, hätte im vorliegenden Band durchaus Erwähnung verdient. Auch Erasmus von Rotterdam und die von ihm beeinflussten „Sakramentarier“, die sich wie Luther von 
der katholischen Kirche trennten, doch Luthers Abendmahlslehre von der „Realpräsenz" Christi ablehnten, spielen in unserem Rezensionsband kaum eine Rolle. Die Einigkeit in der Abkehr von der Papstkirche führte frühprotestantische Kreise keineswegs zu Toleranz als einer evangelischen Tugend. Vielmehr verstärkte das Ringen um die Wahrheit des Evangeliums Abspaltung und Zwietracht. Im Fokus des Rezensionsbandes stehen daher Gelehrte, deren Schriften entweder eine besondere staatsrechtliche oder religiöse Auffassung Richtung Toleranz erkennen lassen oder aus konfessioneller Sicht als „häretisch“ galten.

Toleranz, Duldung, wurde in den religiösen Kämpfen des 16. Jahrhunderts ein Thema jener religiösen Minderheiten, die auf Duldung angewiesen waren, um ihren Glauben leben zu können. Toleranzforderungen erhielten besonderes Gewicht, wenn sie mit biblischen Begründungen versehen werden konnten. So stellt Winfried Schröder die 1605 veröffentlichte Schrift „De libertate religionis" des ungarischen Humanisten Andreas Dudith (1533-1589) vor, die ausgesprochen moderne Züge aufweist, indem das freiheitliche, plurale Verständnis von Religion theologisch begründet wird: Erstens habe der Schöpfer die Menschen darüber im Unklaren gelassen, auf welche Weise er verehrt werden will, woraus sich die auf der ganzen Welt vorhandene religiöse Vielfalt erkläre. Zweitens würde ein völlig einheitlicher Glaube Gottes Freude an der Vielfalt, wie sie sich in der Schöpfung widerspiegelt, nur mindern. Dem Wettstreit, der die Fülle der verschiedenen Weisen der Gottesverehrung ermöglicht, wäre der Boden entzogen, so dass jeder die Freiheit haben sollte, sich in den Fragen der Religionsausübung auf seinen eigenen Verstand zu besinnen. Das sind Argumente, wie sie ähnlich Immanuel Kant in seiner Schrift „Was ist Aufklärung“ 1785 und der Pragmatist William James in seinem Essay „The Will to Believe“ 1897 verwandten. Dudith gab sein katholisches Bischofsamt auf. Er heiratete und wechselte zum (calvinischen) Protestantismus. Seine Schrift enthält Gedanken, die von dem paganen, in Konstantinopel lebenden Gelehrten Themistios aus dem vierten Jahrhundert stammen. Doch Dudiths Gedanken blieben, was ihm durchaus bewusst war, reine Illusion in Bezug auf ihre Verwirklichung in jener Zeit, in der ihr Verfasser lebte.

Vielfach wurde von Toleranz nur im abwertend pejorativen Sinn gesprochen, wie Mona Garloff für die konfessionellen Auseinandersetzungen in Frankreich zur Zeit Heinrichs IV. feststellt; hier besaß traditionsgemäß die durch den König geleistete Einheit von Reich, Gesetz und Religion besonderen Rang. Damit aber bedeutete Toleranz, wie sie den Hugenotten 1598 im Edikt von Nantes gewährt wurde, den ersten Schritt zu einem toleranteren christlichen Europa, auch wenn diese Entwicklung durch den Dreißigjährigen Krieg zunächst einen Rückschlag erlitt.

Das Edikt zu Nantes sicherte den religiösen Frieden (um dessentwillen Heinrich IV. mehrfach die Konfession wechselte) nach Jahrzehnten blutiger Auseinandersetzungen im Land: Den Hugenotten wurde die Ausübung ihrer Religion gestattet unter Beibehaltung des Katholizismus als Staatsreligion. Damit war das Ziel kirchlicher Wiedervereinigung nicht völlig unmöglich, wie sie später Ludwig XIV. 1685 durch Widerruf des Edikts auf seine Weise betrieb. So gesehen gewann in den französischen Religionsdiskursen der Gedanke, eine gewisse Toleranz zuzulassen, doch die Erlaubnis mit einer zeitlichen Begrenzung zu versehen, sehr viel größeres Gewicht als die konfessionelle Annäherung. Dem religiösen Anliegen übergeordnet blieb die Sorge um die politische Einheit des Staates.

Jean Bodin konnte als bedeutendster Staatstheoretiker seiner Zeit in Frankreich und darüber hinaus erfolgreich sein, da er mit seinem Hauptwerk, den sechs Büchern über die Republik, von 1576, als Verfechter des monarchischen Absolutismus den Staatszweck gegenüber der Religion höherstellte. Der Papst setzte seine Werke auf den Index verbotener Bücher. Bis heute ist unklar, ob Bodin, der sich als juristischer Ratgeber für Hexenprozesse einen Namen machte, in seiner Grundeinstellung aber mäßigend und pragmatisch dachte, ein Verfechter des Toleranzgedankens war, 
wurde er doch (wohl zu Unrecht) schon zu seiner Zeit als Verfasser der 1583 erschienenen Schrift, "Colloquium Heptaplomeres“, betrachtet - eine der frühesten Toleranzschriften, die in Form einer Diskussion von sieben Weltweisen auf dem Boden der natürlichen Theologie für Duldung plädierte. Ihre literarische Form erinnert an die erwähnte Schrift über den Glaubensfrieden von Nikolaus von Kues (De pace fidei) nach der Eroberung Konstantinopels durch das Osmanische Reich. Beide Schriften machen den Einfluss des Humanismus auf die Toleranzdebatten der Zeit deutlich.

\section{Täufer, Antitrinitarier und Sozinianer - bibeltreue Häretiker als Feinde von Staat und Kirche}

Üblicherweise zählen im historischen Rückblick Toleranzverfechter zu den wenigen Guten in einer bösen, intoleranten Zeit. Dass dabei die katholische Kirche und die Jesuiten im Urteil heutigen Toleranzdenkens besonders schlecht abschneiden, angesichts ihres - unter Einsatz fragwürdiger Mittel - durchaus erfolgreichen Bemühens, um Wiedergewinnung verloren gegangenen Terrains im Zuge der sogenannten Gegenreformation, muss nicht betont werden. Doch das Bild stimmt nicht ganz. Richtig ist: Als Ausdruck größter Häresie galten die religiösen Anschauungen jener Gruppen, die sich, - extremer als Luther, Zwingli und Calvin dies praktizierten - allein auf die Heilige Schrift hinsichtlich des wahren Glaubensverständnisses beriefen. Dazu gehörten die damals spöttisch als „Wiedertäufer" bezeichneten Gruppen, die die Kindstaufe ablehnten. Praktiziert wurde die Erwachsenentaufe. Die Täuferbewegung verstand sich als Gewalt ablehnende Bruderschaft Christi und lebte in der apokalyptischen Erwartung der Wiederkehr des Herrn.

Die zweite große Bewegung von Häretikern, die sich von der Kirche unter Berufung auf die Bibel abwandte, bildeten die Unitarier (Antitrinitarier). Sie spielen in vorliegendem Band eine bedeutende Rolle, da aus ihrem Kreis die wichtigsten Schriften zur Toleranz im 16. und 17. Jahrhundert stammen. Sie lehnten das Trinitätsdogma ab. Sie waren zwar nicht wie die als politische Umstürzler betrachteten Täufer durch das Mandat des Speyerer Reichsabschiedes von 1529 reichsweit von Verfolgung und Tötung bedroht, doch ein ähnliches Schicksal erfuhren sie durch territoriale Gerichtsbarkeit. Das Maß von Anfeindung und Bedrohung, das sie zu erdulden hatten, war ungeheuer groß. Die Hinrichtung des aus Spanien stammenden humanistischen Arztes und Theologen Michael Servet 1557 mit Wissen und Willen Calvins vor den Toren Genfs auf Grund seiner antitrinitarischen und theosophischen Anschauungen löste religiöse Erschütterungen in ganz Europa aus. Sie veranlassten den Baseler Arzt Sebastian Castellio, in seinen Toleranzschriften die strafrechtliche Verfolgung von Bürgern um ihres Glaubens willen zu verurteilen.

Castellio wurde ein geistiger Wegweiser für Fausto Sozzini, dessen Anhängerschaft der Sozinianer sich ab der zweiten Hälfte des 16. Jahrhunderts in ganz Europa verbreitete. In Sozzinis posthum 1611 erschienenem „Traktat über die Kirche“ wurde Ungeheuerliches behauptet: Dass die Kirche, da sie lediglich eine Versammlung der Gläubigen sei, sich in heilsnotwendigen Dingen irren könne und Seligkeit auch außerhalb der Kirche erlangbar sei. Sozinianer setzten sich in England nicht nur für die Laienpredigt ein, sondern wandten sich theologisch argumentativ auch gegen den Anspruch der Kirche, an Gottes statt zu sprechen oder als Gottes Mittler aufzutreten. Justin Champion, dessen Beitrag sich den Sozinianern Paul Best, John Fry, John Biddle und John Knowles in England im Zeitraum 1641-1665 widmet, vertritt die These, dass nicht im Antitrinitarismus, sondern im Antiklerikalismus, der sich gegen den göttlichen Anspruch der Anglikanischen Kirche richtete, die historische Leistung der Sozinianer zu sehen ist, der Religionsfreiheit langfristig zum Sieg verholfen zu haben. Man hat das Wort "langfristig“ zu betonen, denn in den sechziger Jahren des 17. Jahrhunderts kam es zu mehreren einschneidenden Gesetzen, welche außerhäusliche religiöse Versammlungen von Dissenters unter Strafe stellte und die Vergabe von öffentlichen Ämtern an das 
Bekenntnis zur Anglikanischen Staatskirche band. Selbst der 1689 von Wilhelm III. erlassene Toleration Act, der für eine gewisse Religionsfreiheit sorgte, befreite diejenigen, die zur Staatskirche in Distanz standen, nicht von dem Nachteil, dann kein öffentliches Amt ausüben zu dürfen.

Der Einfluss des Sozianismus Polens und der Niederlande war in England besonders wirksam. Sascha Salatowsky führt in seinem Beitrag aus, wie der Sozinianer Samuel Przykowsky in der Adelsrepublik Polen-Litauen und ebenso der aus Franken stammende Sozinianer Johann Crell in den Niederlanden, mit dem Eintreten für religiöse Toleranz Einfluss gewannen. Im katholischen Polen konnten auf Grund seiner strukturellen politischen Schwäche reformatorische und sozinianische Bewegungen Fuß fassen, die wiederum von den Jesuiten mit rabiaten, durchaus in ihrem Sinne wirksamen Methoden bekämpft wurden, wenn auch ohne Scheiterhaufen (vgl. den Beitrag von Łukasz Bieniasz). Anders war die Situation in den reformatorisch geprägten Niederlanden. Unter der spanischen Besetzung sah der Zusammenschluss zur Utrechter Union 1579 die individuelle Religionsfreiheit vor (vgl. den Beitrag von Wiep van Bunge). Als Ausnahmeerscheinung im damaligen Europa herrschte in den Niederlanden eine aus politischer Selbstbehauptung geborene Liberalität des Denkens, die auch durch das Fehlen einer staatlichen wie kirchlichen Zentralmacht möglich wurde. Die für den Toleranzgedanken grundlegenden Schriften von Spinoza, Locke und Bayle waren nicht zufällig in den Niederlanden verfasst worden. Muslime, die Luther als Feinde betrachtete (die "grausamen Türken"), fanden nach ihrer Einwanderung in die Niederlande im späten 17. Jahrhundert als Gegner des Katholizismus dort eher ein tolerantes Klima vor als in lutherischen Territorien. Die kulturelle Fremdheit wurde von der Unterhaltungskultur aufgenommen. Doch die Duldung islamischer Einwanderer besaß auch Grenzen. Verständnis fanden Muslime weniger bei der reformierten Geistlichkeit als bei Spinozisten, Sozinianer und Remonstranten.

Die vom reformierten Glauben abweichenden Bekenntnisse hatten es in den Niederlanden trotz verkündeter Religionsfreiheit keineswegs immer leicht, wie van Bunge ausführt. Das gilt für vor allem für die Gruppe der Remonstranten, die Calvins Lehre von der doppelten Prädestination ablehnten. Theologen der Remonstranten wie Simon Episcopus und vor allem Philpp van Limborch (mit seiner „Theologia christiana“) entwickelten in ihren Texten einen Eckpfeiler modernen protestantischen Denkens. Sie betonten, dass die theologische Differenz im Verständnis des rechten Glaubens ein typisches, positiv zu wertendes Element des Protestantismus sei (vgl. den Beitrag von Kęstutis Daugirdas). Alle Toleranzdenker des 16. bis 18. Jahrhunderts bis hin zu Gotthold Ephraim Lessing, dessen Ringparabel das literarische Herzstück aufgeklärten Toleranzdenkens darstellt (vgl. den Beitrag von Friedrich Vollhardt), waren in ihrer Zeit keine „normalen“, der gesellschaftlichen Konvention angepassten Gestalten. Nicht selten verlief ihr Leben im Kontext ihrer Toleranzforderung konfliktreich und risikobehaftet.

\section{Entwicklung von Toleranz im Luthertum der Frühaufklärung}

Im Rezensionsband behandelt Walter Sparn am Beispiel der lutherischen Theologen Johannes Gerhard, Theophil Lessing und Franz Budde die Veränderung des Toleranzverständnisses in der lutherischen Orthodoxie des 17. Jahrhunderts. Der Theologe Johannes Gerhard (1582-1637) empfahl, die Praxis der Toleranzausübung der Klugheit der Staatsführung zu überlassen, wandte sich jedoch gegen jede Vermischung von Calvinismus und Luthertum. Der 1669 erschienene Traktat des Theologen Theophil Lessing (Großvater des Verfassers von „Nathan dem Weisen“) behandelte die Frage, ob der Staat Untertanen einer anderen Religion als der vorherrschenden (im Sinne des Augsburger Religionsfriedens von 1555) dulden sollte. Oder muss man die Abweichler nötigen, die vorgeschriebene Religion anzunehmen bzw. sie im Weigerungsfall bestrafen? 
Th. Lessing sprach sich mit seinem naturrechtlich begründeten Eintreten für die Nichtbestrafung, demnach für Toleranz aus. Im lutherischen Staatskirchentum stellte seine Argumentation, wie Walter Sparn erläutert, etwas Neues dar. So deutlich Lessings antiliberaler Konservatismus hervorstach, indem er dem Staat die Pflicht auferlegte, im Falle erkennbar abweichender Religionsausübung einzugreifen, dominierte doch das Plädoyer für ihre Duldung, sofern die Irrenden im Glauben die Gesetze achten und ansonsten kein Aufhebens machen. Der Glaubensfrieden sei von größerem Nutzen für das Gemeinwohl als Glaubenszwang. Während bei Th. Lessing der Religionsbegriff noch ganz lutherisch konfessionell, d.h. mit dem exklusiven Wahrheitsanspruch versehen war, findet man, wie Sparn fortfährt, im Zeitalter der Frühaufklärung bei dem Theologen Franz Budde (1667-1729), zunächst in Halle, ab 1705 in Jena lehrend, einen Religionsbegriff, der nicht mehr normativ vom exklusiven Wahrheitsanspruch lutherischer Theologie bestimmt wird. Unter dem Einfluss der Naturrechtslehren von Hugo Grotius und Samuel Pufendorf wird eine deskriptivfunktionale Religionsauffassung sichtbar. Die Bedeutung des Naturrechts in der protestantischen Frühaufklärung liegt in der Zurückdrängung theologischer Dogmatik durch den Blick auf den Menschen. Durch seine anthropologische Blickwendung ebnete Budde, wie in Nordamerika zu gleicher Zeit die Quäker, den Weg für die Forderung einer allgemeinen religiösen Toleranz, resümiert Sparn.

Wie groß war die Toleranzbereitschaft Nordeuropas? Jens Glebe-Møller untersucht dies für Dänemark. Sein Fazit: Beherrscht von der lutherischen Staatskirche, habe während des gesamten 17. Jahrhunderts die streng lutherisch-orthodoxe, auf die Augsburgische Konfession ausgerichtete Geistlichkeit in dänischen Territorien (wozu auch Norwegen gehörte), Toleranz kaum zugelassen. Obwohl der Katholizismus das stärkste Element im konfessionellen Feindbild bildete, existierte in Kopenhagen eine katholische Gemeinde, die einerseits mit Hilfe katholischer Gesandtenkreise am Leben erhalten blieb nicht zuletzt dadurch, dass in deren Privatkapellen kirchliche Handlungen vollzogen wurden, unter Leitung von kirchenpolitisch aktiven Jesuiten. Einer von ihnen, Heinrich Kircher, musste 1673 auf Grund seiner subversiven Aktivitäten Dänemark verlassen. Auf der anderen Seite war die dänische Krone dort, wo religiöse Toleranz den Handel förderte, nämlich an den Südgrenzen des Staates zum Kontinent hin, bestrebt, sie allen Interessierten kund zu tun, wie Glebe-Møller verdeutlicht. In Glückstadt, 1616 vom dänischen König Christian IV. gegründet, durften sich auch Remonstranten, Mennoniten und sephardische Juden ansiedeln - neben weiteren Glaubensflüchtlngen. Denn Glückstadt sollte als künftige Handelsmetropole in Konkurrenz zu Hamburg treten. 1780 gab der Rendsburger Theologe W.E. Mattiae ein Büchlein heraus „Über die Toleranz in den dänischen Staaten".

Toleranz konnte im Einzelnen sehr Unterschiedliches bedeuten. Galt die Toleranzforderung meist dem Schutz der eigenen Gruppe, so war sie selten darüber hinaus mit dem Ruf nach allgemeiner Toleranz verknüpft, sondern konnte durchaus auf Kosten anderer Gruppen geschehen. Dass aber Toleranz für die eigene Gruppe gegenüber der herrschenden Gewalt ausdrücklich mit der Forderung von Intoleranz gegenüber einer anderen Gruppe gefordert wurde, war eher die Ausnahme. Aber sie gab es, wie der Beitrag von Christian Laursen zeigt. Im Mittelpunkt steht der von einer Herrscherdynastie aus den Völkern der Anden abstammende - hoch gebildete - Felipe Guaman Poma de Ayala (über den auch ein Wikipedia-Eintrag informiert, zum Teil mit von Laursens Befunden abweichenden Deutungen). Er schrieb die erste Geschichte Perus unter spanischer Herrschaft nach der Unterwerfung der Inka (die seit Jahrhunderten über andere Völker Südamerikas geherrscht hatten) und der Zwangschristianisierung der indigenen Bevölkerung. Obwohl Guaman Poma die Grausamkeiten spanischer Kolonialherrschaft kritisierte, solidarisierte er sich mit der Kirche und der spanischen Krone. An König Philipp II. richtete er die Bitte, dieser möge das eigene Volk - Guaman Poma stammte von der Dynastie der Yarovilca ab - gegenüber den Inkas besserstellen, nachdem die eigenen Vorfahren in der Zeit vor der spanischen Eroberung den Inkas dienen 
mussten. Doch als das ursprüngliche Herrschergeschlecht - so die kühne These - stammten die Yarovilca von (,weißen“) Söhnen des biblischen Adam ab, und sie haben nach Meinung Guama Pomas quasi christlich in vorchristlicher Zeit gelebt. Seine Forderung war, die Ethnien in Peru rassisch voneinander zu trennen, um ihre Kultur rein zu erhalten. Das Motiv, den spanischen König zur Apartheid-Politik zu motivieren (den diese Botschaft wohl niemals erreichte), ist nicht schwer herauszufinden: Es ging dem Bittsteller darum, den Einfluss der Inkas, welche die kollektive Erinnerung an die Kulturleistungen der von ihnen Unterworfenen auslöschten, zu begrenzen.

\section{Probleme der Umsetzung rechtlich verankerter Toleranz}

Wenden wir uns zum Schluss noch historischen Beispielen zu, die die Schwierigkeiten der Umsetzung ausgehandelter und vertraglich festgelegter Toleranzgewährung in der Praxis beleuchten. Das Referat von Wolfgang Forster widmet sich dem zu London 1604 geschlossenen Friedensvertrag zwischen England und Spanien nach zwölf aufreibenden Jahren des Seekrieges, der für beide Seiten ruinös war. Wenn England eine Toleranzgarantie für seine Schiffs- und Handelsleute bei der Landung an der Küste Spaniens zur Vertragsbedingung machte, um sie dem Zugriff der Inquisition zu entziehen; dann konnte der zu diesem Zugeständnis gezwungene spanische König Philipp II. nicht erwarten, dass der Inquisitionsausschuss diesem Ansinnen begeistert zustimmte. Musste der spanische König doch auch auf das tief katholisch geprägte Leben des Landes Rücksicht nehmen, ebenso auf das Interesse des Papstes, England zu rekatholisieren. Schwerste Strafe hatte gewöhnlich derjenige zu erwarten, der, auch als Ausländer, das Allerheiligste bei der öffentlichen Fronleichnamsprozession nicht mit Ehrfurcht grüßte, doch das war von einem zufällig anwesenden nichtkatholischen Engländer kaum zu erwarten. Die Lösung derartiger Probleme und die Praxis der Handhabung von Konflikten, die tatsächlich auftraten, stellt der Beitrag von Wolfgang Forster anschaulich dar.

Gewissensfreiheit in Glaubensfragen erfolgte in theologischen und staatsrechlichten Diskussionen selten, ohne zugleich Grenzen oder Ausnahmen der empfohlenen Toleranz anzudeuten - so auch im Letter Concerning Toleration, von John Locke (1689). Die Gewährung von Toleranz war nie nur religiös begründet, sondern verknüpft mit politischen und ökonomischen Motiven. Oft spielten dabei die durch Heirat oder Erbfolge veränderten Konfessionslagen der Herrschaftsdynastie eine Rolle. Toleranzbereite Herrscher konnten auf erbitterten Widerstand ihrer Kirche stoßen - wie im vorliegenden Band das Beispiel Heinrich IV. (von Navarra) in Frankreich belegt. Ähnliches lässt sich aber auch in England aufzeigen. Die Toleranzbereitschaft des Stuart-Königs Karl II. bei seinem Regierungsantritt 1660 stieß auf erbitterten Widerstand der anglikanischen Kirche. Sie lehnte in ihrem Kampf gegen Katholiken, puritanische Dissenters und eingewanderte Lutheraner jede Toleranz ab. Der Katholizismus war für die Anglikanische Kirche der Hauptfeind Nummer eins. Ein toleranzbereiter katholischer König musste bei der hohen Geistlichkeit den Verdacht wecken, die Rekatholisierungspläne Roms zu unterstützen. Nach dem Tod von Elisabeth I. (1603) ging die dynastische Erbfolge an die katholische Stuart-Linie über, die nach der Hinrichtung Karls I. (1649) und dem Scheitern der Pläne des Lordprotektors Oliver Cromwell, aus England eine Republik zu machen, mit Karl II. wieder regierendes Herrscherhaus wurde.

\section{Gesellschaftliche Pluralität - Toleranz förderndes oder Toleranz erschwerendes Faktum?}

Man kann generell davon ausgehen, dass die Argumente von Toleranzbefürwortern, wenn es um den Fortbestand der eigenen Konfession ging, nicht weniger von der Sorge um die wahre Religion und den rechten Glauben bestimmt war als die Haltung der - staatliche oder kirchliche Macht re- 
präsentierenden - Toleranzgegner. Töricht wäre, die christliche Religion ab dem Zeitalter des Humanismus nur als Hemmschuh des Fortschritts auf dem Weg zur Moderne in Richtung Menschenrechte zu betrachten. Vielmehr bot sich dem Christentum in seinen konfessionellen Ausprägungen und der ihnen innewohnenden Dynamik die Chance, diesen Weg im Wechsel politischer Verhältnisse langfristig mit zu gestalten.

Harald Stockert beschreibt in seinem Beitrag die religiösen Gruppen und ihr Zusammenleben unter dem Aspekt der Toleranz in der zweiten Hälfte des 17. Jahrhunderts für Mannheim. Bis 1607 ein Dorf, wurde der Ort mit Privilegien ausgestattet, um calvinische Zuwanderer anzuziehen und wirtschaftliche Prosperität zu erreichen. Im Dreißigjährigen Krieg zerstört und entvölkert stieg Mannheim im Zuge der Wiederansiedlungspolitik unter dem kurpfälzischen Regenten Karl Ludwig rasch zur „Vielnationenstadt“ auf. Es war durch seine zu Karrees angeordneten Häuserblocks als Quadrate-Stadt schon damals überregional bekannt. Doch berühmter wurde Mannheim als Metropole des Zusammenlebens eines bunten Gemischs verschiedener Volks- und Glaubensgruppen. Darunter befanden sich Sozinianer und Täufer. Die Stadt war bis zum Herrscherwechsel 1685 reformatorisch geprägt, darüber hinaus besaß sie eine Multikonfessionalität wie kein anderer Ort Europas. Neben Lutheranern und Katholiken existierte eine Vielzahl kleinerer religiöser Gruppen, die nicht nur aus dem Reich, sondern aus vielen Ländern Europas zugezogen waren, dem Aufruf des Kurfürsten folgend, der das ausgeblutete Land ökonomisch aufbauen wollte. Die Gruppen von Reformierten blieben als wallonische (bzw. französische), niederländische (niederdeutsche) und hochdeutsche Gemeinde jeweils für sich, ohne eine Vereinigung anzustreben. Sie dominierten den Rat und die Politik der Stadt. Zwischen ihnen herrschte ein gemeinsames Bewusstsein, der richtigen Religion anzugehören, aber es gab eine Reihe von Konflikten. Anlass dazu boten etwa die streng gehandhabte Kirchenzucht, die Mischehen und vor allem die gerechte Verteilung der Almosengelder. Das Verhältnis zu anderen Glaubensgruppen war stark unterkühlt und konfliktanfällig, auch wenn eine gewisse Pragmatik im Umgang vorherrschte. So sahen sich Lutheraner benachteiligt, weil ihnen lange Zeit, bis 1673, weder ein eigener Geistlicher noch öffentliche Gottesdienste erlaubt waren. Die Lutheraner, deren Auftreten in der Öffentlichkeit für die reformierte Geistlichkeit ein Ärgernis blieb, hofften auf ein eigenes Kirchengebäude. Doch der Kurfürst, der eine Annäherung der Konfessionen wollte, entschied, dass das Gebäude als Kirche simultan von allen drei Konfessionen zu benutzen sei. Das brachte die Katholiken in besondere Schwierigkeiten, da ihnen auch verboten wurde, den Empfang der Sakramente in katholischen Kirchen benachbarter Territorien wahrzunehmen. Die Situation änderte sich, nachdem 1685 der katholische Philipp Wilhelm die Nachfolge von Karl Ludwig als Regent angetreten hatte. Jetzt verloren die Reformierten ihre führende Stellung. Mehr Toleranz brachte diese Wendung nicht.

Das Ergebnis der Analyse Stockerts ist eindeutig. Es war die Politik des Kurfürsten, der die Stadt zwang, eine "tolerante“ Kommunalpolitik zu betreiben, die zahlreiche Konflikte beinhaltete. Das galt insbesondere für die in größerer Zahl ansässigen Juden, denen die großen Konfessionen intolerant begegneten. Doch der Regent schätzte sie als Wirtschaftsfaktor. Mit Recht warnt Stockert davor, das unterstellte "friedliche Zusammenleben“ im historischen Mannheim als MulitkultiHarmonie zu betrachten und sie zum Vorbild zu machen für die heute geforderte Toleranz gegenüber Migranten und Flüchtlingen. Dass ein solches konfliktreiches Zusammenleben überhaupt möglich war, verdient allerdings festgehalten zu werden.

Am Ende stellt sich die Frage: Sind Migranten toleranter? Alexander Schunka entlarvt in seinem Beitrag zunächst einen Mythos, der in der protestantischen Migrationsgeschichtsschreibung eine gewisse Rolle spielt. Da wurden die frühneuzeitlichen Flucht- und Wanderungsbewegungen aus religiösen Gründen gern dargestellt als „Resultat konfessioneller Intoleranz und Engstirnigkeit 
weltlicher und kirchlicher Obrigkeiten in den Ausgangsländern“, welcher Einhalt geboten wurde durch die „mildtätige Ansiedlungspolitik einzelner, wahrhaft christlicher Fürsten“ (Schunka). Der Autor zeigt, welches Interesse protestantische Herrscher hatten, die Pflege eines solchen Narrativs den eigenen Geschichtsschreibern zur Pflicht zu machen. Derartige Mythen schienen auch geeignet zu sein, die Vaterlandsliebe nachfolgender Generationen zu stärken, wenn sie in Lesebüchern und im Geschichtsunterricht ausgebreitet werden. Katholische Herrscher verfügten ebenfalls über interessengeleitete Narrative, die die Treue zur allein seligmachenden Kirche beschworen. Man hat zur Kenntnis zu nehmen, so Alexander Schunka, dass die religiös motivierten Migrationsbewegungen in der frühen Neuzeit nicht punktuelle Ereignisse waren, sondern sehr viel häufiger den Strukturen einer seit längerem vorhandenen geographischen Mobilität folgten. Schunka macht darauf aufmerksam, dass die politischen Forderungen von Exulanten sich keineswegs auf die allgemeine Freistellung individueller Bekenntniszugehörigkeit richteten. Vielmehr ging es ihnen um die Herstellung eines in der Erinnerung ideal überhöhten Zustandes früherer Zeit, in der der Glaube derart unbehelligt ausgeübt werden konnte, wie dies die Erinnerungsverklärung suggerierte. Religiöse Autonomie zu fordern war ein Mittel, die Bedrückung der eigenen Gruppe zu lindern und die materielle Versorgung der betroffenen Familien zu sichern - nicht mehr. Das heißt, Autonomie für alle wurde eher für schädlich gehalten. Schunka belegt diese These am Beispiel von differenten Migrantengruppen aus Böhmen, die in mehreren Orten Kursachsens im 17. Jahrhundert angesiedelt wurden. Die nicht nur religiösen, sondern auch ethnischen und sprachlichen Differenzen ließen das Bedürfnis erkennen, die eigene Identität durch scharfe Abgrenzung von anderen Gruppen zu stärken. Im Ergebnis wurde alles andere als die heute moralisch so hoch bewertete Offenheit für Toleranz gefördert, sondern ein „exulantisches Sonderbewusstsein“ kultiviert, welches der leidensvollen eigenen Erfahrung die Hartherzigkeit anderer Gruppen gegenüberstellte. Das Leiden für die Reinheit des eigenen Glaubens gewann als Narrativ die Funktion, den Gemeinschaftswillen zu stärken - eine durchaus funktional-pragmatische Einschätzung. Schunka betont, dass die kommunalen Ämter und Behörden meist überfordert waren, einerseits den Anordnungen der fürstlichen Kanzlei, andererseits der Masse der Migranten bei nur bescheiden vorhandenen Ressourcen gerecht zu werden.

Das Resümee dieses aufschlussreichen Essays lautet, dass Pluralität im gesellschaftlichen Zusammenleben nicht etwas ist, das per se zu Toleranz führt, wie heute in der hohen Wertschätzung einer pluralen Gesellschaft offenbar naiv angenommen wird. Pluralität ist vielmehr bloßes Faktum, das zu akzeptieren die betroffenen Gruppen erst bereit sind, wenn sie gelernt haben, im eigenen Interesse mit Glaubenszwietracht umzugehen. Diese Erkenntnis, zu der man als Leser des vorliegenden Bandes gelangt, erinnert den Rezensenten an einen Zeitungsartikel von Jens Bisky in der „Süddeutschen Zeitung (Nr. 278, S. 13) vom heutigen Tage, dem 1. Dezember 2016, an dem diese doch recht ausführlich gewordene Buchbesprechung zum Abschluss kommt. Bisky schreibt: „Die freie Gesellschaft muss lernen, mit ihren Feinden umzugehen." In der Tat. Wer sich heute für Toleranz in konkreten Konfliktsituationen einsetzt, wird dies kaum mit der Forderung nach Frieden, Wertschätzung und Humanität erreichen, die sowieso jeder mit sich herumträgt. Vielmehr hat man Machtverhältnisse und Interessenlagen mitsamt ihren Kontexten zu analysieren, um pragmatische Lösungswege zu finden, muss dabei aber auch zu erwartende Konfliktlagen in Rechnung stellen.

\section{Einsichten}

Aus der Realgeschichte des 16. bis 18. Jahrhunderts, wie sie sich im vorliegenden Band in einzelnen Facetten zeigt, lässt sich unter anderem lernen, dass gewährte Toleranz damals sehr viel weniger dem Drang christlicher Nächstenliebe folgte (obwohl sie nie völlig ausgeschlossen war), sondern aus einem Prozess des Abwägens der politisch Verantwortlichen hervorging. Die Erhaltung der 
bestehenden Ordnung und der bestehenden Religion als dem wichtigsten stabilisierenden Element kirchlicher wie staatlicher Herrschaft stand dabei im Vordergrund der Politik römisch-katholischer Herrscherdynastien. Ihr Interesse war der Ausbreitung des Toleranzgebotes eher hinderlich. Monarchische Herrschaft erwartete in der frühen Neuzeit die Dienstbarkeit des Volkes und dessen Unterordnung unter die Religion des Herrschers. Das Sonderleben einzelner Gruppen mit einem eigenen religiösen Wahrheitsanspruch duldete die Kirche nicht. Unterschiedliche Bekenntnisse blieben ein Unruheherd auch für die weltliche Ordnung, insbesondere wenn das Zusammenleben in dichten Siedlungsgebieten zu ständiger Konfrontation herausforderte. Dezentralisierte, schwächer strukturierte Regierungssysteme und geringere Siedlungsdichte verminderten religiöse Konflikte, aber sie führten, wie das Beispiel der Besiedlung Amerikas durch europäische Glaubensflüchtlinge zeigt, nicht automatisch zu größerer Toleranz; die Siedlergemeinschaften besaßen bis zur amerikanischen Unabhängigkeitserklärung 1776 den Charakter von Theokratien. Die in Europa im Namen des „rechten“ Glaubens geführten Kriege, in denen man jeweils Andersgläubige als Feinde verfolgte, folterte und tötete, wurden auf dem Boden bestehender religiöser Intoleranz geführt. Die Zerstörungen, die der Krieg hinterließ, boten der Toleranz Chancen dank einer umsichtigen Ansiedlungspolitik.

Die Ideen zu einem modernen Toleranzbegriff kamen nicht von den Repräsentanten der herrschenden Mächte, sondern von einzelnen Gelehrten, die das Schicksal teilten, religiöser oder politischer Intoleranz ausgesetzt zu sein. Aufgeklärte Monarchen bedienten sich ihrer. Die negativen Konnotionen des Toleranzbegriffs aus der Perspektive einer das Volk unterwerfenden Herrschaft unter Kirche und Krone bedurften einer positiven Umfunktionierung. Sie beruhte auf einem im Zuge der Aufklärung entdeckten Einsicht, dass das Ziel der Vervollkommnung des Menschseins und der Besserung darniederliegender sozialer Verhältnisse nur im Medium eines persönlichen Raumes der Freiheit möglich ist, die selbstverantwortliches Handeln stärkt. Eine gute und gerechte Regierung erkennt den sehr viel größeren Nutzen einer in freiwilliger Bindung erfolgten, von bürgerlichem Gemeinsinn getragenen Zustimmung zur vorherrschenden Ordnung, als dies bloße Unterwerfung unter das Gehorsamsgebot leisten könnte. Das religiöse Bekenntnis sollte demnach nicht mehr von weltlich-kirchlicher Gewalt erzwungen werden, sondern persönlich-privater Entscheidung überlassen sein.

Der vorliegende Band regt an zu einer neuen Diskussion über Toleranz im historischen Kontext. Es erscheint zweifelhaft, ob die heute unter dem Begriff der Menschenwürde gefasste wechselseitige Respektierung der Person - unabhängig von ethnischen Merkmalen und religiösen Bindungen über den Toleranzbegriff voll erschließbar ist. Religiöse Toleranz impliziert die Anerkennung eines Anspruchs auf ein Erscheinungsbild in der Öffentlichkeit, das der Ausübung des tolerierten Bekenntnisses entspricht. Doch wie Diskussionen um nicht verletzende sprachliche Termini oder um das Tragen von Kopftuch und Burka zeigen, sind hier zahlreiche Konflikte möglich, die die Frage des wechselseitig Zumutbaren berühren. Die einseitig gewährte, von humanen Grundsätzen getragene Toleranz lebt von der Hoffnung auf einen Gewinn für beide Seiten. Die Toleranz gewährende ethnische Mehrheit bzw. deren Regierung räumt einer auf Duldung angewiesenen Minderheit Rechte ein, auch unter Inkaufnahme von Risiken. Die Staatsleitung hofft, dabei sowohl pragmatische Vorteile für das gesellschaftliche Wachstum zu gewinnen, als auch höheren Zielen wie Frieden, Humanität und Verständnisbereitschaft gerecht zu werden. In der Regel betonen die Führungseliten des Gemeinwesens durchaus, höherwertigen Zielen verpflichtet zu sein, wenn mit der Toleranzgewährung konkrete Erwartungen in Bezug auf ihren Nutzen für das gesamtgesellschaftliche Wohl erfolgreich umzusetzen sind. Die auf Toleranz angewiesene Minderheit wird eigene Vorstellungen vom guten Leben in der sie aufnehmenden Gesellschaft an die hier vorfindbaren Bedingungen anzupassen haben, indem sie sowohl Chancen nutzt, als auch erkennbare Einschränkungen und 
Grenzen zu beachten lernt.

Als Wert, der zur Menschenwürde gehört, wird Toleranz ihrem normativen Anspruch nicht dadurch gerecht, dass sie den Menschen, die auf sie angewiesen sind, unter dem Deckmantel einer pluralen Gesellschaft das Dauerschicksal sozialer Zweitklassigkeit beschert. Politische Toleranz impliziert aufs Ganze gesehen die volle Teilhabe an den bürgerlichen Rechten, Pflichten und Lebenschancen in dieser Gesellschaft. Dahinter verbergen sich in einer auf engem Raum lebenden Gesamtbevölkerung mit multikulturellem Gepräge Prozesse kultureller Anpassung, die selten konfliktfrei sind. Die Lernprozesse finden auf beiden Seiten statt, nur - und das macht den Begriff „wechselseitig" unscharf - gelernt wird im positiven Fall weitgehend Unterschiedliches während im negativen Fall, bei verfehlter Annäherung, Enttäuschung auf beiden Seiten vorfindbar ist - verursacht durch Konflikte, die entweder auf die unzureichende Realisierung gewährter Toleranz für eine um Aufnahme nachsuchende Minderheit, auf fehlende Toleranzbereitschaft innerhalb der Bevölkerungsmehrheit oder auf eine noch allzu schwach entwickelte kulturelle Lernbereitschaft der emigrierten Minderheit zurückgehen.

Man erkennt, dass eine ideengeschichtliche Betrachtung, welche „Erlaubnistoleranz“ ganz unten im Toleranzranking ansetzt und „hohe wechselseitige Wertschätzung“ zum Gipfel der Werteordnung einer auf Pluralität wie auf Anteilnahme beruhenden modernen Gesellschaft erklärt, sich jenseits der aus der Geschichte gewonnenen Erfahrungen befindet. Nicht ohne sarkastische Färbung sagt einem heutige Lebenserfahrung, dass die vielfach geforderte Toleranz in unserer liberal-pluralen Gesellschaft dort erwartbar ist, wo sie nicht von der Befürchtung lebt, dass sie einen selbst oder der eigenen (ethnischen, religiösen oder sozialen) Bezugsgruppe etwas kosten könnte, demnach mit materiellen oder ideellen Einschränkungen verbunden ist. In der pluralen Gesellschaft ist „Wechselseitigkeit" sozialer Akzeptanz nur als Prinzip, doch kaum als Realbeziehung möglich, in der jeder mit jedem verbunden ist, auch wenn Handy und Internet, Blogging und Twittern, einen solchen Verbund ermöglichen würden. Der mediale Sozialverbund hat leider auch neue Formen von Intoleranz geschaffen, genannt Cybermobbing. Praktisch jedoch sind, wie sozialpsychologische Befunde bestätigen, unseren Möglichkeiten, mit anderen Menschen in persönlichen, wertebildenden Kontakt zu treten, quantitative Grenzen gesetzt. Nachhaltige Kommunikation vollzieht sich durch Selbstbegrenzung der daran beteiligten Personen in Form einer Gruppe. Es ist problematisch, denjenigen das Prinzip der Wechselseitigkeit aufzubürden, die von der Gewährung einer einseitig ausgesprochenen Bereitschaft leben, als mittellose Minderheit von der ökonomisch starken Mehrheit akzeptiert zu werden. Nach christlicher, nicht zuletzt paulinischer Ethik, setzt wechselseitiger Respekt einseitige Bereitschaft voraus, zumindest zeitweise Einschränkungen hinzunehmen: „Einer trage des anderen Last", schrieb Paulus an die Galater (Gal 6,2). Die Forderung ist nicht pluralistisch relativierbar, sondern geht alle an. Sie verlangt ein gemeinsames Bekenntnis zu humanem Handeln.

Im Alltag dominiert heute ein Begriff von Toleranz, der wechselseitigen Respekt in der kommunikativen Beziehung meint - ironisch gesagt, dazu auffordert, sich wechselseitig im Ertragen der Macken des Anderen zu üben. Dieses Verständnis von Toleranz setzt die Gleichheit sozialer Machtverhältnisse der an der Situation Beteiligten voraus. Sind sie ungleich, was eher die Regel als die Ausnahme darstellt, wird die Forderung wechselseitiger Duldung zynisch: Es wäre Hohn, von einem „Neuen“ in der Klasse Duldung zu fordern für den Fall, dass er dem aggressiven Spott einer Clique von Klassenkameraden ausgesetzt ist, die behauptet, die dunkle Hautfarbe des Neuzuganges nur ertragen zu können, wenn der Neue ihre Art des Umgangs mit ihm ebenfalls dulde. Dass nach den Verbrechen des 20. Jahrhunderts und der Missachtung der Menschenrechte in heutigen Kriegszonen „wechselseitige Wertschätzung“ im Umgang der Menschen miteinander eine unabdingbare 
Forderung ist, bleibt davon unberührt. Davon ist jene einseitige Toleranz, die Verfolgten Lebenschancen eröffnet, zu unterscheiden. Um sie geht es hier. Toleranz im politischen Sinne setzt ein Machtgefälle voraus, das nicht durch eine auf Gleichheit beruhende Wertung aufhebbar ist. Bezogen auf vergangene Jahrhunderte lässt sich heute durchaus von größerer Toleranzbereitschaft in den westlichen Demokratien sprechen. Zugleich macht die aktuelle Diskussion um Begrenzung der Zuwanderung von Gruppen, die inhumanen Lebensbedingungen in ihrer Heimat zu entkommen suchen, die Sensibilität des Themas Toleranz deutlich.

Die Verankerung der Religionsfreiheit als Grundrecht in den liberalen Demokratien der Gegenwart geht über die Gewährung religiöser Toleranz aufgeklärter Fürsten früherer Jahrhunderte weit hinaus. Sie ist zurückzuführen einerseits auf eine durch den Säkularisierungsprozess veränderte gesellschaftliche Rolle der christlichen Religion, andererseits auf die wachsende Fähigkeit zur Selbsteinsicht des durch die Aufklärung hindurchgegangenen Menschen der Moderne. Dabei ist praktizierte Toleranz im Alltag als Tugend eines auf Empathie und Freundlichkeit beruhenden Zusammenlebens keineswegs überflüssig geworden.

Schon länger verzichten die großen Konfessionen darauf, die Botschaft des Evangeliums im Sinne des augustinischen „Compelle intrare“ zu verstehen. Auch wenn es nicht weiter betont wurde, siegte die Bemühung, den Zeichen der Zeit zu folgen. Vom Begriff „Judenmission“ trennte sich die Evangelischen Kirche Deutschlands (EKD) erst auf ihrer Synode in Magdeburg, am 9. November 2016, in Form einer öffentlichen Erklärung. Katholische und evangelische Kirche arbeiten heute an ihrem Erscheinungsbild. Sie verstehen ihre Botschaft als Einladung an alle Menschen, Gläubige wie Nichtgläubige. Mit ihren sozialen Diensten, mit seelsorgerischer Betreuung, mit einem integrativen, von der Haltung der „Annahme“ bestimmten Gemeindeleben sowie mit öffentlichen Stellungnahmen zu kontroversen Zeitfragen nimmt die Kirche zentrale Aufgaben in der modernen Gesellschaft wahr und findet in dieser Funktion auch allgemeine Akzeptanz. Das gilt ebenso für ihre Mitwirkung bei der Bestimmung eines gesellschaftlich relevanten, praktisch umsetzbaren Toleranzbegriffs.

\section{Literatur}

Salatowsky, Sascha \& Schröder, Winfried (Hrsg.) (2016): Duldung religiöser Vielfalt - Sorge um die wahre Religion. Toleranzdebatten in der Frühen Neuzeit. Friedenstein-Forschungen. Hrsg. v. Forschungszentrum Gotha für kultur- und sozialwissenschaftliche Studien der Universität Erfurt. Band 10. Stuttgart: Franz Steiner (313 Seiten; ISBN 978-3-515-11368-7; 313 Seiten; Preis: 56.00 Euro (Deutschland); eBook-Ausgabe: ISBN 978-3-515-11369-4).

\section{Über den Autor}

Prof. em. Dr. Hein Retter: Technische Universität Braunschweig, Institut für Erziehungswissenschaft (Deutschland). Website: www.tu-braunschweig.de/allg-paed/personal/ehemalige/hretter. Kontakt: h.retter@tu-bs.de 